Int. J. Morphol.,

31(4):1198-1204, 2013

\title{
Característica de la Composición Corporal de Agresores y Víctimas de Bullying
}

\author{
Characteristics of the Body Composition of Aggressors and Victims of Bullying
}

Levandoski, G. \& Cardoso, F. L.

LEVANDOSKI, G. \& CARDOSO, F. L. Característica de la composición corporal de agresores y víctimas de bullying. Int. J. Morphol., 31(4):1198-1204, 2013.

RESUMEN: El objetivo fue verificar la composición corporal de escolares agresores y víctimas de bullying. Participaron en el estudio 184 alumnos de sexo masculino con media etaria de 12,77 años pertenecientes a una escuela de enseñanza pública de la ciudad de Florianópolis, Brasil. Fueron utilizados el Cuestionario para el Estudio de Violencia entre Pares, un examen socio-métrico, observación y el somatotipo. El análisis estadístico fue realizado a través del programa SPSS versión 14.0, con nivel de significancia fijado en p $<0,05$. La incidencia de alumnos partícipes en bullying fue 28,3\%, donde 14,1\%, 4,3\% y 9,8\% eran víctimas, agresores/víctimas y agresores, respectivamente. En relación a la clasificación del somatotipo, las víctimas obtuvieron clasificación endomórfica mesomorfo; los agresores/víctimas como mesomorfo equilibrado; y los agresores como endomórfico mesomorfo. Se encontraron evidencias, en términos antropométricos, de que los alumnos agresores presentaron mayores índices de IMC con predominio de masa corporal magra.

PALABRAS CLAVE: Bullying; Composición corporal; Somatotipo; Niños y adolescentes; Educación física.

\section{INTRODUCCIÓN}

En la actualidad, uno de los temas que viene despertando la curiosidad y el interés de profesionales de las áreas de educación y salud en todo el mundo es el fenómeno del bullying escolar (Levandoski \& Cardoso, 2010 y 2013).

El bullying puede ser definido como un conjunto de actitudes agresivas, intencionales y repetitivas que ocurren sin motivación evidente, adoptado por uno o más alumnos contra uno o varios, causando dolor, angustia y sufrimiento terrible a las víctimas, generando como consecuencias, bloqueos psicológicos (Olweus, 1994). Actualmente, según Smith (2004), otro concepto muy utilizado en los estudios es la definición de Rigby (2002) como "abuso sistemático de poder".

La prevalencia del bullying aún es un asunto polémico y diversos estudios indican diferentes valores. Así se han obtenido diferentes tasas de victimización como, por ejemplo, de 15\% (Kim, 2004); 16\% (Lemeshow et al., 2008; Fekkes et al., 2005); $18 \%$ (Pellegrini et al., 1999); 21,4\% (Carvalhosa et al., 2001); 27\% (Storch et al., 2007); 28\% (Griffiths et al., 2006); 39,8\% (Wolke et al., 2001) con alumnos en edad escolar y 17\% (Rivers, 2004); $21 \%$ (Wolke \&
Sapouna, 2007) evaluando adultos que retrataron valores recordados de victimización en el período escolar.

Las personas que son víctimas de bullying cuando niños, son más propensas a sufrir de bloqueos psicológicos y, a lo largo de los años, aumentan las probabilidades de tornarse agresores envueltos en la criminalidad Freire et al. (2006) debido a la marginalización que ya reciben tanto en la escuela como por la sociedad. Según Rodríguez Piedra et al. (2006), existen algunos estudios que demostraron una pequeña relación entre perturbaciones mentales en la edad adulta con el sufrimiento de victimización durante la infancia o adolescencia. En este sentido, algunas víctimas, en su proceso de crecimiento, se tornaron adultos agresivos, pues presentaron la convicción de que la agresividad es la mejor forma de alcanzar sus objetivos.

En Brasil, la somatotipología enfatiza la caracterización de perfiles específicos de atletas en diversas modalidades deportivas clasificándolos en cuanto a su función o posición en la táctica dentro del equipo deportivo, tanto como, facilitando la búsqueda y selección de talentos deportivos (Fernandes Filho, 2003). La mayoría de los estudios aborda

\footnotetext{
"Docente, Universidade Federal do Vale do São Francisco (UNIVASF), Petrolina-PE, Brasil.

** Centro de Ciencias de la Salud y del Deporte (CEFID), Docente del Doctorado en Ciencias del Movimiento Humano, Universidad del Estado de Santa Catarina (UDESC), Santa Catarina, Brasil.
} 
este tipo físico relacionándolo con el desempeño de atletas de diferentes deportes colectivos e individuales (Rivera Sosa, 2006; Carter et al., 2005; Levandoski et al., 2007). El estudio del somatotipo se constituye en un valioso instrumento de información que permite la visualización global de las modificaciones morfológicas ocurridas en función de alteraciones procesadas simultáneamente en nivel de los tejidos muscular, óseo y adiposo (Guedes \& Guedes, 1999).

Según Guedes \& Guedes, pocos estudios fueron realizados con la intención de proporcionar nuevas informaciones que contribuyan al proceso de crecimiento y de maduración de individuos en poblaciones de no atletas. De esta forma, nuestros objetivos fueron más allá de saber si la predicción de un individuo está adecuada a los patrones preestablecidos por los investigadores de esta área. Se deseó utilizar estas estrategias para confirmar las hipótesis de que los alumnos partícipes en casos de bullying eran realmente los niños más fuertes/débiles, altos/bajos, delgados/gordos o si los alumnos con mayor aptitud física eran los agresores, puesto que, muchas veces, los alumnos víctimas poseen poca vivencia de prácticas motoras en las clases de educación física, que muchas veces proporcionan la selección y exclusión de niños a partir de sus habilidades individuales, propiciando un escenario para que los alumnos con mayor estatus construyan su identidad encima de los de menor estatus formando así sus relaciones de poder y jerarquía dentro del grupo.

Se creyó que las variables que miden la composición corporal a partir de técnicas antropométricas podían indicar diferencias entre los actores que actúan como agresor y víctima en el proceso del fenómeno bullying. Siendo así, este trabajo tuvo como objetivo verificar la composición corporal de los escolares agresores y víctimas de bullying en la ciudad de Florianópolis, Brasil.

\section{MATERIAL Y MÉTODO}

Participaron de este estudio 184 alumnos del sexo masculino con media etaria de 12,77 años regularmente matriculados en $6^{\circ}$ básico de educación primaria, seleccionados de forma intencional de una escuela de enseñanza pública de la ciudad de Florianópolis, Brasil. La elección de estos cursos para la realización de la evaluación se debió al hecho de que las acciones de este fenómeno, son más evidentes entre alumnos con edades entre 11 a 13 años (Lyznicki et al., 2004).

Con el objetivo de verificar a los alumnos con características de bullying fueron utilizados el Cuestionario para el Estudio de Violencia entre Pares de Freire et al., un examen socio-métrico construido a partir de los estudios (Freire et al.; Smith; Pereira, 2002; Olweus) y la observación informal realizadas por los pesquisidores (en sala de clase, recreo, en los corredores durante los intervalos de clase, en las clases de educación física y en la salida de clases). De acuerdo con Wolke \& Sapouna (2007) los instrumentos utilizados para identificar las víctimas y los agresores de bullying son, generalmente, cuestionarios que analizan seis formas de victimización administradas metodológicamente de forma retrospectiva de la infancia o actual (en la adolescencia). Los seis puntos clave deben corresponder a la retrocedencia del hecho, pertenencias dañadas, agresión verbal, el hecho de que las víctimas sean usadas como objeto de diversión, exclusión social e historias. Levandoski \& Cardoso (2013), retrataron aún, que una gran limitación era acreditar que los instrumentos proporcionaran un resultado sin error, o que indicaran fielmente los alumnos con características de agresores o de víctimas.

Las variables antropométricas mensuradas fueron masa corporal, estatura, envergadura, circunferencia de brazo (BR), pierna (PA), cintura (CI), cadera (QD), tanto como diámetro biepicondiliano del húmero (U) y diámetro biepicondiliano del fémur (FE). También se midieron el espesor de los pliegues cutáneos (tríceps (TR), subescapular (SB), suprailíaca (SI), supraespinal (SE), abdomen (AB), pantorrilla medial (PM) (Guedes, 1994).

El cálculo del porcentaje de grasa corporal (\%GC) fue obtenido a partir del Protocolo de Slaughter para niños y adolescentes entre 7 a 18 años en nivel de madurez postpubertad. Los cálculos para la obtención de la masa corporal magra (MM), masa corporal gorda (MG), relación cintura-cadera (RCQ), índice de masa corporal (IMC) fueron realizados utilizando el procedimiento de (Fernandes Filho). El somatótipo fue determinado siguiendo método antropométrico propuesto por Carter \& Heath (1990).

Los valores recogidos fueron organizados y analizados en el Statistical Package for the Social Sciences (SPSS, versión 14.0) for Windows, con nivel de significancia fijado en $p<0,05$. Se utilizó estadística descriptiva para indicar el porcentaje de frecuencia de los partícipes en el fenómeno bullying. Para verificar si los datos presentaban distribución normal fue efectuado el test de Shapiro-Wilk. Confirmando la normalidad, se realizó el test de ANOVA con Tukey post hoc para indicar la diferencia entre los grupos y correlaciones con coeficiente de Pearson.

La dirección de la escuela fue informada sobre los procedimientos que serían utilizados y los alumnos relacionados participaron de forma voluntaria, donde sus respon- 
sables firmaron el Acuerdo de Consentimiento Libre y Esclarecido. La pesquisa fue realizada de acuerdo con la Resolución 196/1996 del Consejo Nacional de Salud y aprobada por el Comité de Ética en Seres Humanos de la Universidad del Estado de Santa Catarina.

\section{RESULTADOS}

En la Tabla I, es presentada la proporción y porcentaje de los alumnos de sexo masculino que manifestaron o no participación en acciones de bullying, tanto como la media etaria por grupo de participación. Se encontró una tasa de 28,3\% de partícipes en el fenómeno bullying. A través de estos alumnos, se descubrió que no existe un lugar específico o pre-determinado para la realización de estas prácticas entre pares, porque ellas acontecieron durante las clases, en los corredores, baños, recreos y en los intervalos. Por lo tanto, en esta pesquisa, no se abordó factores ligados al diagnóstico sobre la incidencia del fenómeno, pero si se analizó y comparó las diferencias corporales de los partícipes en este fenómeno escolar.
La Tabla II, presenta los factores asociados a los alumnos partícipes en el fenómeno bullying a partir de sus variables de la composición corporal. En la referida tabla son descritos los valores promedios y desviaciones estándar, así como el resultado del test de análisis de varianza (ANOVA) utilizando el Post Hoc de Tukey con valor de $\mathrm{p}<0,05$.

Tabla I. Participación en el fenómeno bullying.

\begin{tabular}{lccc}
\hline Categorías & n & Frecuencia & Media \\
\hline Víctima & 26 & 14,1 & 12,23 \\
Agresor/víctima & 8 & 4,3 & 12,75 \\
Agresor & 18 & 9,8 & 13,61 \\
Sin participación & 132 & 71,7 & 12,49 \\
Total de alumnos & 184 & & \\
Total de alumnos & 52 & 28,3 & 12,77 \\
partícipes & & & \\
\hline
\end{tabular}

Fueron encontradas diferencias estadísticamente significativas en las variables masa corporal, estatura, envergadura, masa corporal magra (MM), circunferencia de brazo (Circ. BR) y de pierna (Circ. PA). Los alumnos agresores presentaron mayores proporciones corporales en relación a los alumnos víctimas y agresores/víctimas. La variable diá-

Tabla II. Variables de la Composición Corporal entre los alumnos partícipes en bullying.

\begin{tabular}{|c|c|c|c|c|c|}
\hline Variables & $\begin{array}{c}\text { Víctima } \\
\mathrm{n}=26 \\
\text { Media (dp) }\end{array}$ & $\begin{array}{c}\text { Agr./Vict. } \\
\quad \mathrm{n}=8 \\
\text { Media (dp) }\end{array}$ & $\begin{array}{c}\text { Agresor } \\
\mathrm{n}=18 \\
\text { Media (dp) }\end{array}$ & $\mathbf{F}$ & $\mathbf{P}$ \\
\hline Masa & $45,96 \pm 13,89 a$ & $43,33 \pm 9,54^{a}$ & $56,08 \pm 12,36^{\mathrm{b}}$ & 4,225 & $0,020 *$ \\
\hline Estatura & $1,54 \pm 0,09^{a}$ & $1,53 \pm 0,10^{a}$ & $1,62 \pm 0,09 \mathrm{~b}$ & 3,494 & $0,017 *$ \\
\hline Envergadura & $1,55 \pm 0,12$ a & $1,55 \pm 0,06^{\mathrm{a}}$ & $1,66 \pm 0,09 \mathrm{~b}$ & 6,179 & $0,004 *$ \\
\hline IMC & $19,01 \pm 4,29$ & $18,15 \pm 2,19$ & $21,19 \pm 3,73$ & 2,388 & 0,152 \\
\hline Sumatoria de 6 Pliegues & $80,23 \pm 44,28$ & $67,25 \pm 26,41$ & $90,67 \pm 47,57$ & 0,841 & 0,437 \\
\hline$\% \mathrm{G}$ & $19,94 \pm 11,76$ & $16,14 \pm 6,12$ & $21,10 \pm 11,53$ & 0,566 & 0,572 \\
\hline MG & $10,49 \pm 10,50$ & $7,05 \pm 2,81$ & $12,80 \pm 9,93$ & 1,022 & 0,367 \\
\hline MM & $35,47 \pm 5,41$ a & $36,28 \pm 8,59 a$ & $43,28 \pm 6,96 \mathrm{~b}$ & 8,107 & $0,001 *$ \\
\hline RCQ & $0,83 \pm 0,05$ & $0,81 \pm 0,03$ & $0,83 \pm 0,04$ & 0,455 & 0,714 \\
\hline Endomorfia & $3,91 \pm 01,85$ & $3,34 \pm 1,28$ & $4,20 \pm 1,85$ & 0,650 & 0,527 \\
\hline Mesomorfia & $4,51 \pm 1,47$ & $4,39 \pm 0,79$ & $4,91 \pm 1,16$ & 0,662 & 0,521 \\
\hline Ectomorfia & $3,16 \pm 2,01$ & $3,40 \pm 1,24$ & $2,76 \pm 1,70$ & 0,426 & 0,655 \\
\hline Circ. BR & $23,86 \pm 3,84 \mathrm{a}$ & $23,12 \pm 3,55 a$ & $26,61 \pm 3,07 \mathrm{~b}$ & 3,171 & $0,026 *$ \\
\hline Circ. PA & $31,26 \pm 4,43 a$ & $31,50 \pm 3,92^{a}$ & $34,69 \pm 3,62 b$ & 3,213 & $0,024 *$ \\
\hline Circ. CI & $68,50 \pm 11,42$ & $65,62 \pm 4,65$ & $73,00 \pm 8,13$ & 1,450 & 0,230 \\
\hline Circ. QD & $82,03 \pm 10,06$ & $80,75 \pm 6,38$ & $87,55 \pm 8,84$ & 1,722 & 0,164 \\
\hline Diám. BR & $6,47 \pm 0,49$ & $6,29 \pm 0,03$ & $6,77 \pm 0,52$ & 2,297 & 0,079 \\
\hline Diám. FE & $9,52 \pm 0,53$ & $9,43 \pm 0,41$ & $9,59 \pm 0,52$ & 0,325 & 0,807 \\
\hline Diám. OM & $32,92 \pm 0,02 a$ & $33,63 \pm 0,02$ & $35,06 \pm 0,02 \mathrm{~b}$ & 2,698 & $0,047 *$ \\
\hline Pliegue TR & $14,27 \pm 6,94$ & $12,38 \pm 4,86$ & $13,94 \pm 7,01$ & 0,269 & 0,848 \\
\hline Pliegue SB & $11,38 \pm 7,60$ & $8,75 \pm 2,96$ & $13,06 \pm 7,00$ & 0,955 & 0,415 \\
\hline Pliegue SI & $11,23 \pm 7,15$ & $8,75 \pm 3,65$ & $14,39 \pm 7,92$ & 1,505 & 0,215 \\
\hline Pliegue SE & $11,08 \pm 7,71$ & $9,00 \pm 4,62$ & $13,94 \pm 8,83$ & 1,084 & 0,357 \\
\hline Pliegue AB & $17,42 \pm 10,56$ & $16,13 \pm 8,00$ & $20,44 \pm 12,12$ & 0,448 & 0,719 \\
\hline Pliegue PA & $14,85 \pm 7,19$ & $12,25 \pm 4,59$ & $14,89 \pm 6,72$ & 0,555 & 0,646 \\
\hline
\end{tabular}

$* \mathrm{p}<0,05$ 
metro del hombro (Diám. OB) apuntó las mismas diferencias, por lo tanto, el grupo de alumnos agresores/víctima no se diferenció de las demás categorías.

Las demás variables descritas no se mostraron diferentes para los grupos de participación en bullying. Entre éstas, se encuentran la mayoría de las variables que contribuyen para calcular las componentes del somatótipo. El somatótipo es un instrumento de información que permite la visualización global de las modificaciones morfológicas de la estructura corporal. A través de él, son expresados tres valores numéricos que representan los componentes físicos expuestos siempre en el mismo orden: endomorfia, mesomorfia y ectomorfia. Con el resultado se percibió que no existe una relación directa entre los tres componentes del somatotipo y los alumnos partícipes en bullying de este estudio. No se pudo afirmar que no existan diferencias en el somatótipo entre las tres categorías de bullying estudiadas, sólo inferir que en esta etapa de madurez no fue constatada diferencia entre los participantes del estudio. Se hace hincapié en la importancia de esta averiguación también en diferentes edades de crecimiento y desarrollo.

En la Figura 1, se puede visualizar la somatocarta de los valores encontrados, donde las víctimas obtuvieron clasificación endomórfica mesomorfa; los agresores/víctimas clasificados como: mesomorfo equilibrado; y los agresores clasificados como: endomórfico mesomorfo.

Otra variable que no se diferenció en términos estadísticos fue el índice de masa corporal IMC. A pesar de que

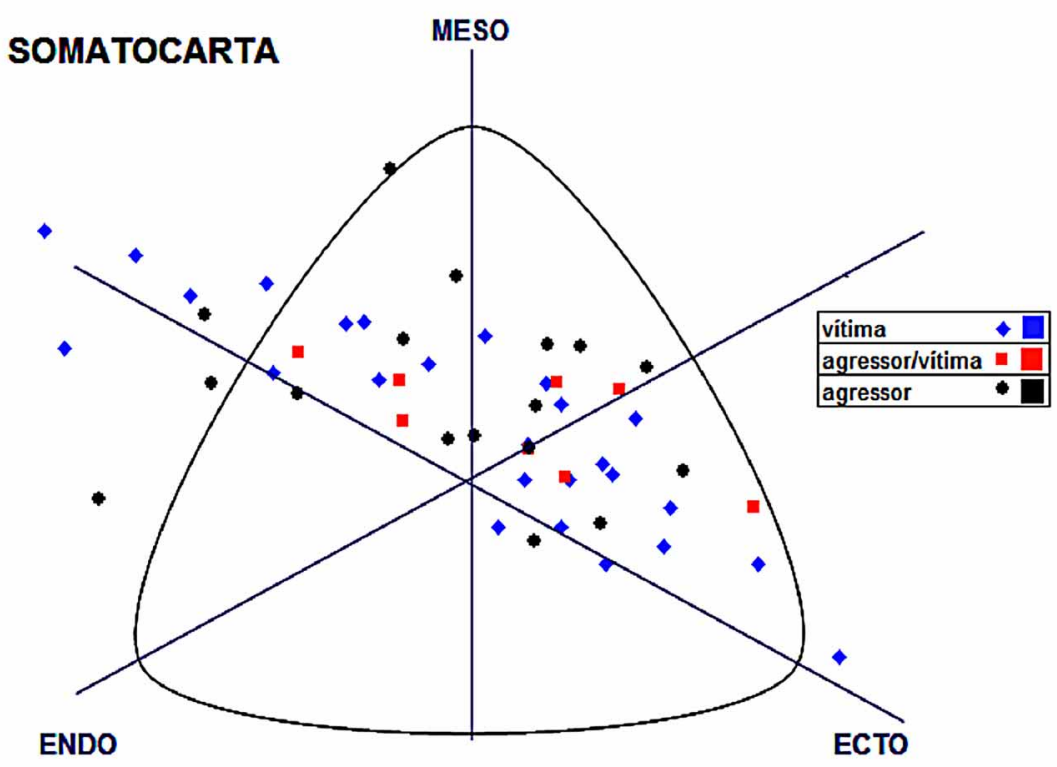

Fig. 1. Somatocarta con los valores de los somatótipos de los alumnos partícipes en bullying. los agresores mostraron índices de IMC más elevados, el que indica que pueden estar con una masa corporal excedente, no se pudo afirmar cuál es la cantidad de masa corporal magra y gorda. Al compararse los índices de masa corporal magra (MM) y gorda (MG) de los agresores y de las víctimas, se percibió que el mayor IMC de los agresores puede ser explicado por el mayor índice de masa magra, el que constituyó diferencia significativa en relación a las víctimas. De esta manera, se sugiere que la diferencia en relación al tamaño corporal de los individuos partícipes en bullying no está en la cantidad de grasa, sino que en el mayor peso del tejido muscular.

\section{DISCUSIÓN}

La obesidad infantil es considerada un problema de salud pública (WHO, 2000) y también un factor que predispone la participación de bullying en niños y niñas (Griffiths et al.). Algunos estudios (Crosnoe et al., 2008; Lemeshow et al.; Verplanken \& Velsvik, 2008; Lunde et al., 2006; Janssen et al., 2004; Smolak, 2004; Strauss \& Pollack, 2003; Shelton \& Liljequist 2002; Odea \& Caputi, 2001) con individuos de origen norte-americano, donde son registrados los mayores índices de obesidad infantil (Lissau et al., 2004), asocian problemas relacionados a la imagen corporal y las relaciones de estatus entre los individuos con IMC sobre el percentil 85. Sin embargo, para efecto de comparación, no se encontraron, en este estudio, individuos con valores sobre o próximos a esta referencia. Entre los factores que sirvieron de hipótesis para explicar el motivo de no haber encontrado alumnos con señales de obesidad, lo más adecuado era pensar en la localización geográfica donde viven los participantes, pues se trataba de una región litoral de fuerte economía pesquera, lo que puede proporcionar a los moradores una alimentación balanceada con nutrientes saludables y frescos. En segundo lugar, pensando en la diversidad del estatus socio-económico de estos alumnos (Sabbag, 2008), oriundos de escuela pública, se pudo partir de la hipótesis de Bracco et al. (2002), relatando que el bajo nivel socio-económico pudo estar asociado a la desnutrición.

Confirmando esta hipótesis, Lemeshow et al. (2008) creyeron que el IMC proporciona una buena refe- 
rencia de la forma corporal en las diversas fases de la vida. Por lo tanto, en este estudio, se descubrió que el IMC no indicó diferencia en relación a la participación de bullying, a pesar de las variables que constituyen la fórmula del IMC (masa y estatura) apuntaran diferencia entre estos grupos de participación.

Los individuos con IMC elevados están siempre sujetos a situaciones de victimización, bajo estatus y autoestima (Janssen et al.), pero se observó una relación inversa frente al IMC. Los alumnos agresores fueron los que presentaron IMC más elevado, pero sin indicar señales de sobrepeso. Así una de las grandes contribuciones de este estudio, diferenciándolo de los demás mencionados, es que se buscó estrategias que analizaran la composición corporal de los alumnos partícipes en bullying con técnicas más precisas. El IMC indicó que los individuos pudieron estar con una masa corporal excedente, pero no afirmar cuál es la preponderancia de masa corporal magra o gorda. Así, se descubrió que la diferencia de la composición corporal de los individuos partícipes en bullying no estaba en la cantidad total de masa corporal gorda (MG), pero si en la cantidad de masa corporal magra (MM), de acuerdo a los valores presentados en la Tabla II.

En el estudio de Needham \& Crosnoe (2005), la masa corporal no estuvo asociada a los síntomas de depresión entre los niños, pero los resultados de este estudio apuntaron a que las víctimas y los agresores/víctimas presentaron masa corporal total y una peor auto-evaluación sobre la imagen corporal menor que los alumnos agresores. No se usaron mecanismos para controlar los síntomas de depresión, pero con la utilización de la escala de la silueta corporal de Stunkard et al. (1983), se encontraron diversas correlaciones negativas solamente entre las víctimas y la insatisfacción con la imagen corporal y las siguientes variables: $r=-0,653 p=0,004$ masa corporal total; $r=-0,774 p<0,001$ IMC; $r=-0,718 p=0,001$ sumatoria de los pliegues; $r=-0,641 \mathrm{p}=0,006 \mathrm{MG} ; \mathrm{r}=-0,550 \mathrm{p}<0,022$ RCQ; $r=-0,773 p<0,001$ endomorfia; $r=-0,836 p<0,001$ mesomorfia; $r=-0,926 p<0,001$ auto-imagen percibida; por lo tanto, cuanto mayores eran los valores de estos marcadores morfológicos de las proporciones corporales, menor fue la satisfacción con la imagen corporal.

En relación a la imagen corporal ideal, en promedio, se percibió que, tanto las víctimas como los agresores/ víctimas gustarían de ser mayores físicamente, pero no fueron encontradas correlaciones entre estas variables. Dos víctimas poseían exceso de peso, pero no presentaban índices de obesidad, y ambas gustarían de ser menores físicamente.

\section{CONCLUSIONES}

Se cree que las variables cineantropométricas ejercieron influencia entre los autores que participaron en las acciones en el fenómeno bullying. Siendo así, este trabajo tuvo como objetivo verificar la composición corporal y somatotipo de escolares agresores y víctimas de bullying en una edad específica.

La literatura indicó que individuos con masa corporal total elevada presentaban mayor incidencia a transformarse en víctimas de bullying, hecho no corroborado en este estudio. Sin embargo, cuando se analizó la composición corporal a través de procedimientos antropométricos, se notó que los agresores poseían una masa corporal magra significativamente mayor.

Así a partir de los resultados, se constataron fuertes evidencias en términos antropométricos, de modo que los alumnos agresores presentaban aspectos morfológicos superiores en relación a las víctimas.

LEVANDOSKI, G. \& CARDOSO, F. L. Characteristics of the body composition of aggressors and victims of bullying. Int. J. Morphol., 31(4):1198-1204, 2013.

SUMMARY: The study objective was to verify the school corporal composition of bullying victims and aggressors. A total of 184 boys with an average age of 12.77 years from a public school in the city of Florianópolis took part in this study. We used the Questionnaire for the Study of the Violence among Equal, a sociometric exam, observation and the somatotype. The statistical analysis was accomplished through the program SPSS, version 14.0, with significance level of $\mathrm{p}<0.05$. The incidence was $28.3 \%$ of students involved in bullying, being 14.1, 4.3 and 9.8\% were victims, aggressors/victims and aggressors, respectively. In relation to classification of the somatotype the victims obtained classification as endomorphic/mesomorphic; the aggressors/victims as balanced mesomorphic; and the aggressors as endomorphic/mesomorphic. We found evidences in anthropometric terms that the aggressor boys present larger indexes of IMC with prevalence of thin corporal mass.

KEY WORDS: Bullying; Corporal composition; Somatotype; Children and adolescent; Physical education. 


\section{REFERENCIAS BIBLIOGRÁFICAS}

Bracco, M. M.; Ferreira, M. B. R.; Morcillo, A. M.; Colugnati, F. \& Jenovesi, J. Gasto energético entre crianças de escola pública obesas e não obesas. Rev. Bras. Ciên. e Mov., 10(3):29-35, 2002.

Carter, J. E. L. \& Heath, B. H. Somatotyping: development and applications. New York, Cambridge University Press, 1990.

Carter, J. E.; Ackland, T. R.; Kerr, D. A. \& Stapff, A. Somatotype and size of elite female basketball players. J. Sports Sci., 23(10):1057-63, 2005.

Carvalhosa, S. F.; Lima, L. \& Matos, M. G. Bullying: A provocação/vitimação entre pares no contexto escolar português. Aná. Psicológica, 19(4):523-537, 2001.

Crosnoe, R.; Frank, K. \& Mueller, A. S. Gender, Body Size and Social Relations in American High Schools. Soc. Forces, 86(3):1189-216, 2008.

Fekkes, M.; Pijpers, F. I. M. \& Verloove-Vanhorick, S. P. Bullying: who does what, when and where? Involvement of children, teachers and parents in bullying behavior. Health Educ. Res., 20(1):81-9, 2005

Fernandes Filho, J. A Prática da Avaliação Física. 2a ed. Rio de Janeiro, Shape, 2003.

Freire, I. P.; Veiga Simão, A. M. \& Sousa Ferreira, A. O estudo da violência entre pares no $3^{\circ}$ ciclo do ensino básico: um questionário aferido para a população escolar portuguesa. Rev. Port. de Educação, 19(2):157-83, 2006.

Griffiths, L. J.; Wolke, D.; Page, A. S.; Horwood, J. P. \& ALSPAC Study Team. Obesity and bullying: different effects for boys and Girls. Arch. Dis. Child., 91(2):121-5, 2006.

Guedes, D. P. Composição Corporal: princípios, técnicas e aplicações. 2a ed. Londrina, APEF, 1994.

Guedes, D. P. \& Guedes, J. E. R. P. Somatótipo de crianças e adolescentes do município de Londrina-Paraná-Brasil. Rev. Bras. Cineantropom. Desempenho Hum., 1(1):7-17, 1999.

Janssen, I.; Craig, W. M.; Boyce, W. F. \& Pickett, W. Associations between overweight and obesity with bullying behaviors in school-aged children. Pediatrics, 113(5):1187-94, 2004.

Kim, S. J. A Study of Personal and Environmental Factors Influencing Bullying. Dissertação de Mestrado- Psicologia. Münchem, Germane, 2004.

Lemeshow, A. R.; Fisher, L.; Goodman, E.; Kawachi, I.; Berkey, C. S. \& Colditz, G. A. Subjective social status in the school and change in adiposity in female adolescents: Findings from a prospective cohort study. Arch. Pediatr. Adolesc. Med., 162(1):23-8, 2008.

Levandoski, G. \& Cardoso, F. L. Body image and social status of Brazilian students involved in bullying. Rev. Latinoam. Psicología, 45(1):135-45, 2013.

Levandoski, G. \& Cardoso, F. L. Percepção docente sobre as relações de agressividade, o lúdico e o "bullying" na escola. Pensar Prát., 13(2):1-13, 2010.

Levandoski, G.; Cardoso F. L. \& Cieslak, F. Perfil somatótipo, variáveis antropométricas, aptidão física e desempenho motor de atletas juvenis de voleibol feminino da cidade de Ponta Grossa/PR. Fit. Perf. J., 6(5):309-14, 2007.

Lissau, I.; Overpeck, M. D.; Ruan, W. J.; Due, P.; Holtein, B. E.; Hediger, M. L. \& Health Behaviour in School-aged Children Obesity Working Group. Body mass index and overweight in adolescents in 13 European Countries, Israel, and the United States. Arch. Pediatr. Adolesc. Med., 158(1):27-33, 2004.

Lunde, C.; Frisén, A. \& Hwang, C. P. Is peer victimization related to body esteem in 10-year-old girls and boys? Body Image, $3(1): 25-33,2006$.

Lyznicki, J. M.; Mccaffree, M. A. \& Robinowitz, C. B. Childhood Bullying: Implications for Physicians. Am. Fam. Physician, 70(9):1723-8, 2004.

Needham, B. L. \& Crosnoe, R. Overweight status and depressive symptoms during adolescence. J. Adolesc. Health, 36(1):48$55,2005$.

Odea, J. A. \& Caputi, P. Association between socioeconomic status, weight, age and gender, and the body image and weight control practices of 6- to 19- year-old children and adolescents. Health Educ. Res., 16(5):521-32, 2001.

Olweus, D. Bullying at school: basic facts and effects of a school based intervention program. J. Child. Psychol. Psychiatry, 35(7):1171-90, 1994.

Pellegrini, A. D.; Bartini, M. \& Brooks, F. School bullies, victims, and aggressive victims: Factors relating to group affiliation and victimization in early adolescence. J. Educ. Psychol., 91(2):216-24, 1999.

Pereira, B. O. Para uma escola sem violência: estudo e prevenção das práticas agressivas entre crianças. Lisboa, Fundação Calouste Gulbenkian, 2002.

Rigby, K. A meta-evaluation of methods and approaches to reducing bullying in pre-schools and in early primary school in Australia. Canberra, Commonwealth Attorney-General's Department, 2002. 
Rivera Sosa, J. M. Valoración del somatotipo y proporcionalidad de futbolistas universitarios mexicanos respecto a futbolistas profesionales. Rev. Int. Med. Cienc. Act. Fís. Deporte., 6(21):16-28, 2006.

Rivers, I. Recollections of bullying at school and their long-term implications for lesbians, gay men, and bisexuals. Crisis, 25(4):169-75, 2004.

Rodríguez Piedra, R.; Seoane Lago, A. \& Pedreira Massa, J. L. Niños contra niños: el bullying como trastorno emergente. An. Pediatr. (Barc), 64(2):162-6, 2006.

Sabbag, S. Estereótipos de gênero na percepção e avaliação do desenvolvimento motor de meninos e meninas. Dissertação de Mestrado-Ciência do Movimento Humano, Florianópolis, Universidade do Estado de Santa Catarina, 2008.

Shelton, S. \& Liljequist, L. Characteristics and behaviors associated with body image in male domestic violence offenders. Eat. Behav., 3(3):217-27, 2002.

Smith, P. K. Bullying: Recent Developments. Child Adolesc. Ment. Health, 9(3):98-103, 2004.

Smolak, L. Body image in children and adolescents: where do we go from here? Body Image, 1(1):15-28, 2004.

Storch, E. A.; Milsom, V. A.; Braganza, N.; Lewin, A. B.; Geffken, G. R. \& Silverstein, J. H. Peer victimization, psychosocial adjustment, and physical activity in overweight and at-riskfor-overweight youth. J. Pediatr. Psychol., 32(1):80-9, 2007.

Strauss, R. S. \& Pollack, H. A. Social Marginalization of Overweight Children. Arch. Pediatr. Adolesc. Med., 157(8):746-75, 2003.

Stunkard, A. J.; Sorenson, T.; Schlusinger, F. Use of the Danish Adoption Register for the study of obesity and thinness. In: Kety, S. S.; Rowland, L.P.; Sidman, R. L. \& Matthysse, S. W. (Eds.). The genetics of neurological and psychiatric disorders. New York, Raven, 1983. pp.115-20.

Verplanken, B. \& Velsvik, R. Habitual negative body image thinking as psychological risk factor in adolescents. Body Image, 5(2):133-40, 2008.

World Health Organization. Obesity: Preventing and managing the global epidemic. Geneva, World Health Organization, 2000.

Wolke, D. \& Sapouna, M. Big men feeling small: Childhood bullying experience, muscle dysmorphia and other mental health problems in bodybuilders. Psychol. Sport Exer., 9(5):595-604, 2008.

Wolke, D.; Woods, S.; Bloomfield, L. \& Karstadt, L. Bullying involvement in primary school and common health problems. Arch. Dis. Child., 85(3):197-201, 2001.

\author{
Dirección para Correspondencia: \\ Prof. Dr. Gustavo Levandoski \\ Doutor em Educação Física - UFPR \\ Mestre em Ciência do Movimento Humano - UDESC \\ Universidade Federal do Vale do São Francisco (UNIVASF) \\ Petrolina - PE \\ BRASIL
}

Email: glevandoski@gmail.com

Recibido : 29-08-2012

Aceptado: 10-08-2013 